\title{
Evaluation of flexibility in hybrid WDM/TDM PONs
}

\author{
Abhishek Dixit, Bart Lannoo, Goutam Das, Didier Colle, Mario Pickavet, Piet Demeester \\ Department of Information Technology, Ghent University - IBBT, B-9050 Gent, Belgium \\ abhishek.dixit@intec.ugent.be
}

\begin{abstract}
Introducing a WDM dimension on the top of a TDM PON system is a natural evolution to increase the capacity of an optical access network, and this can also offer additional flexibility options. Several hybrid WDM/TDM PON architectures can be designed, each with a different degree of flexibility, going from fully static, over partially flexible to fully flexible architectures. The more flexible architectures, however, are either more expensive, experience a higher power loss or are less secure. A question that arises is if a fully flexible architecture is really needed, or if a partially flexible architecture already can serve several flexibility advantages, like energy efficiency, network migration and network extensibility. An important assessment parameter is the number of needed wavelengths at a certain network load. For a fully flexible architecture, it is clear that the number of needed wavelengths can be optimally minimized, but how big is the gain of a fully flexible architecture compared to a partially flexible one, and what is the minimum degree of flexibility required to have a significant advantage of the offered flexibility. In this paper we evaluate through simulation different architectural options for a hybrid WDM/TDM PON, in terms of flexibility. For this purpose, we use an underlined well-suited medium access control (MAC) protocol that exploits the offered flexibility in terms of dynamic wavelength allocation.
\end{abstract}

Keywords: optical access, PON architectures, hybrid WDM/TDM, flexibility, dynamic bandwidth allocation

\section{INTRODUCTION}

Currently, telecom operators are adapting their broadband access networks for offering highly demanding services such as high-definition TV, interactive gaming, video-conferencing, etc. Optical fiber networks are considered the most future-proof next generation access (NGA) technologies. Nowadays, the most used optical fiber access network configuration is a (power splitting) time division multiplexing (TDM) passive optical network (PON), with Ethernet PON (EPON) and gigabit PON (GPON) as the two most important standards The currently deployed EPON or GPON systems, however, are unable to provide the expected residential data rates by the year 2020 , being a sustainable data rate of $500 \mathrm{Mbps}$ per user (or subscriber) and a peak data rate of 1 Gbps per user [1]. Typically these PON systems are using a separate wavelength (of 1 or $2.5 \mathrm{Gbps}$ ) for down- and upstream, and both wavelengths are then shared between multiple users (e.g., 16, $32,64)$. As the users share the same pool of capacity, competition may arise and traffic requests may not be honored due to congestion.

The mentioned capacity bottleneck for TDM PONs is currently tackled by the standardization activities for the $10 \mathrm{G}$ xPON systems (10G EPON and 10G GPON, respectively). The physical access bit rate is pushed to 10 Gbps per wavelength, firstly for the downlink part and secondly in a symmetric offer for the uplink part. Another attractive PON solution is the wavelength division multiplexing (WDM) PON, offering two separate wavelength channels per subscriber. A pure WDM PON provides an individual down- and upstream wavelength channel to each user, and thus there is no competition among them and no congestion will occur in the network. However, there is also no opportunity to share capacity among the subscribers, and to use the available network resources in a flexible way.

Introducing a WDM dimension on top of a TDM PON system combines the increased capacity delivered by WDM and the inherent capacity sharing of a TDM PON, and a hybrid WDM/TDM PON system is an important candidate for nextgeneration optical access (NGOA) networks. The flexibility to offer any bandwidth (in the limit of the physical bit rate) to anybody could be a major advantage for NGOA networks as it gives a better bandwidth usage, scalability and upgradeability than a pure WDM PON. Different hybrid WDM/TDM PON flavors, with varying remote node architectures, are presented in [2]. The architectures presented in [2] vary in terms of flexibility, cost, power consumption, and data security. We refer 'flexibility' as the architectural capability to offer the same network (PON) performance (like propagation delay from user to OLT, packet loss rate etc.) for a smaller number of used wavelengths. We discuss the various advantages and constrains of flexibility in section III. While the aspects like cost and power consumption has been evaluated in [2], the gains of the flexibility are not well studied in the literature. In this paper, we evaluate the gains of flexibility. Our study will help network operators and service providers to choose the most suitable architecture for NGOA networks.

The remainder of this paper is organized as follows. Section II provides the summary of different architectures and their qualitative comparison in terms of security, flexibility and power loss. Section III provides a brief account of the motivation to add flexibility in a hybrid WDM/TDM PON. In section IV, we demonstrate the simulation results to highlight the need for flexibility and provide insights on how much flexibility is required. Finally section $\mathrm{V}$ concludes the paper.

\section{HYBRID WDM/TDM PON ARCHITECTURES}

A general architecture of a (flexible) hybrid WDM/TDM PON is shown in Fig. 1. Three network parts are indicated between the optical line terminal (OLT) in the central office (CO) and the optical network unit (ONU) at the user side: feeder between OLT and remote node 1 (RN1), distribution between RN1 and RN2, and last mile between RN2 and ONU. 


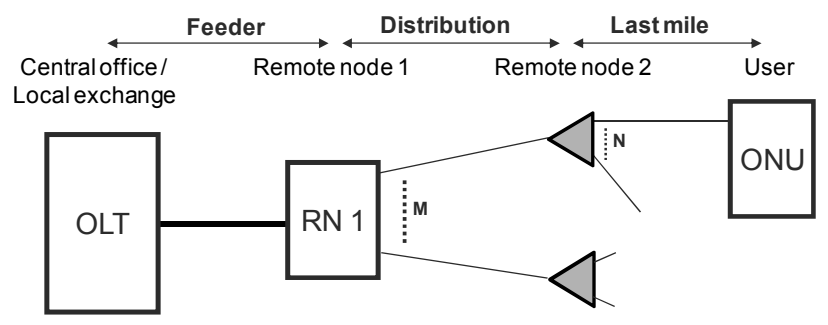

Figure 1. General hybrid WDM/TDM PON architecture

In RN2, a passive power splitter $(1: N)$ is installed, which means that RN1 is connected to $M$ TDM PON architectures. By varying RN1, three main categories of hybrid WDM/TDM PONs are presented in this section, each with a different degree of flexibility: (1) fully flexible, (2) fully static and (3) partially flexible. Choosing the best architecture is typically a trade-off between flexibility on one hand, and cost, power budget and security constraints on the other hand. In this section, a basic assessment for the different architectures is presented, based on the following criteria: flexibility, simplicity (related to cost), power loss (related to power budget) and security.

\section{A. Fully Flexible Architectures}

Table I (a) provides an example of RN1 of a fully flexible architecture, which is referred to in the literature as broadcastand-select (B\&S) hybrid WDM/TDM PON. In a B\&S hybrid WDM/TDM PON, RN1 is a power splitter, and all wavelengths are broadcast to all TDM PONs (or RN2s), and by consequence to all ONUs. In this way, each ONU is time shared with the other ONUs with the advantage of having access to a WDM dimension. In the downstream direction, all wavelength channels are broadcast from the local exchange to all the users, without any selectivity in the network itself. The selection is done by the ONU at the user side. This architecture is very simple (using a legacy power-split optical distribution network) and provides full flexibility. However, it suffers from high power losses and it also has a serious security threat as the

TABLE I. REMOTE NODE 1 VARIANTS FOR (A) FULLY FLEXIBLE (B) FULLY STATIC AND (C) PARTIALLY FLEXIBLE HYBRID WDM/TDM PONS

\begin{tabular}{|l|l|l|l|}
\hline & $\begin{array}{c}\text { (a) B\&S RN1, } \\
\text { with power } \\
\text { splitter }\end{array}$ & $\begin{array}{c}\text { (b) WS RN1, } \\
\text { with AWG }\end{array}$ & $\begin{array}{c}\text { (c) B\&S RN1, } \\
\text { with power splitter } \\
\text { combined with AWG }\end{array}$ \\
\hline & & & \\
& & & \\
& & & \\
\hline Flexibility & Fully flexible & Fully static & Partially flexible \\
\hline Power loss & High & Low & Average \\
\hline Security & Low & High & Average \\
\hline
\end{tabular}

content of all wavelengths is available to all ONUs. Note that a coherent detection technique at the receiver can deal with the high power losses as it allows increasing the optical link budget up to $50 \mathrm{~dB}$ (compared to ca. $30 \mathrm{~dB}$ for direct detection techniques in current $\mathrm{PON}$ architectures). For an access network, however, coherent detection is still a very complicated and expensive technique to be implemented in practical scenarios.

\section{B. Fully Static Architectures}

Table I (b) shows an example of RN1 of a fully static hybrid WDM/TDM PON architecture, referred to as a wavelength-split (WS) hybrid WDM/TDM PON. In this architecture, each wavelength is routed to only one fixed TDM PON (or RN2), and each TDM PON can be reached by only one fixed wavelength. In a WS hybrid WDM/TDM PON, a passive wavelength splitter or filter (e.g., arrayed waveguide grating (AWG)) is put in RN1 to distribute different wavelengths to different TDM PONs, as shown in Table I (b), with $M=W_{u}$ (number of upstream wavelengths) $=W_{d}$ (number of downstream wavelengths). This architecture is also extensively discussed in literature, e.g. [3]-[4]. Note that a separate AWG is depicted for the up- and downlink direction, as typically different wavelength bands are used for upstream (e.g., C-band, 1530 - $1565 \mathrm{~nm}$ ) and for downstream (e.g., Lband, $1565-1625 \mathrm{~nm}$ ). If the same wavelength band is used, a single AWG can be used for both the up- and downlink part. As an AWG has much less power loss compared to a passive power splitter, this architecture has a better power budget and can support more users and a longer reach. Moreover, it also has a high security. However, the flexibility is very restricted as each wavelength is connected to a fixed TDM PON, and this cannot be rearranged with e.g. a changing traffic demand.

\section{Partially Flexible Architectures}

Table I (c) presents an example of a possible RN1 of a partially flexible hybrid WDM/TDM PON architecture. This third category of architectures generally have a higher security and lower power loss than the fully flexible architectures, and are, of course, more flexible than a fully static architecture. Often, a trade-off between these different parameters will decide about the best architecture in a specific situation. In a partially flexible architecture, each TDM PON can be reached by multiple wavelengths and similarly each wavelength can reach multiple numbers of TDM PONs. Note that RN1 shown in Table I (c) makes use of power splitters to offer multicasting. In this way, we termed this architecture as a B\&S hybrid WDM/TDM PON combined with AWG. Here RN1 consists of an AWG followed by multiple $\left(m_{A W G}\right.$ for the uplink and downlink direction, respectively) power splitters, to distribute one wavelength to different TDM PONs, as shown in Table I (c), where $m_{A W G}$ is the number of AWG ports and $m_{s}$ is the number of ports (or TDM PONs connected to) per power splitter in RN1. By using an AWG, the high power splitter loss as compared to a B\&S-PON is reduced by a factor $m_{A W G}$ (assuming that the total RN1 split factor $M=m_{A W G} \times m_{s}$ is kept constant), and only a low insertion loss of e.g. $3 \mathrm{~dB}$ of an $\mathrm{AWG}$ is added. Besides, by including a (smaller) power splitter, some flexibility from a B\&S-PON is still available. This architecture is evaluated in detail in Section IV to estimate the influence of 
flexibility on the total number of used wavelengths and to understand its importance for the PON performance.

\section{Motivation to AdD FleXiBILITY IN ACCESS NETWORKS}

In a hybrid WDM/TDM PON, multiple TDM PONs can be set up from the OLT, each at a specific wavelength. Each TDM PON serves a set of users, and within this set, the capacity is shared. By means of wavelength selection or routing, the number of users within the set can be varied, and thus the capacity offered per user can be varied. Hence a flexible hybrid WDM/TDM PON can offer capacity-on-demand, and the congestion probability can be significantly reduced compared to a static wavelength configuration. In the next subsections, we give a brief account of the three most striking features of a flexible architecture that separate it from most of the other PON architectures, i.e., network extensibility, energy efficiency and network migration. Finally, we also mention the constraints that come with an enhanced flexibility.

\section{A. Network Extensibility}

The network extensibility means the efficient deployment strategies that the hybrid WDM/TDM PON architecture can promote while constantly expanding the network by the incremental installation of network equipment according to the connectivity demand of the end users. Due to the inherent flexibility, the proposed architecture can be used for green- or brown-field installation where the network connectivity demand can change and evolve over time. The example provided in Fig. 2 explains the concept in a more illustrative way.

Fig. 2 provides an example where the first remote node has physically three possible splitting stages. Now, initially as shown in the left part of Fig. 2 the number of users connected to the second and third splitting-point is less than optimal. Therefore due to the wavelength flexibility of our architecture, we can allocate a single wavelength (green for example) to all the users attached to the second and third splitting-point as depicted in Fig. 2 and therefore use just two transceivers and line cards at the OLT. As the demand increases, and more subscribers are added to the relevant splitting-points, the OLT can have added line cards to fulfill the demands of the expanded network, and the wavelengths at the user premises can be reallocated to cope with the individual traffic needs as shown in the right-hand part of the Fig. 2. This provides a means for the operator for a smooth and incremental expansion of the network according to the demand, while keeping the basic network infrastructure deployed in the field undisturbed.
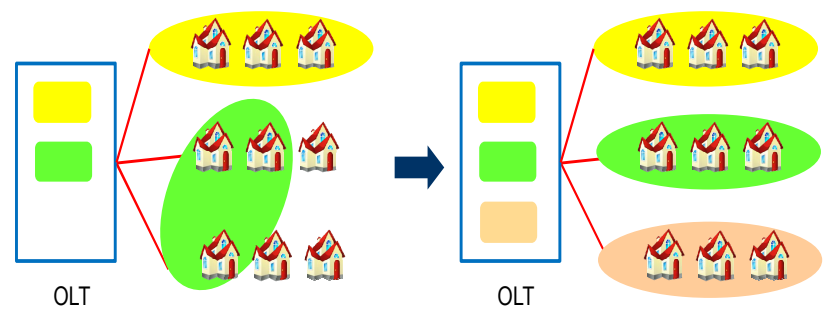

Figure 2. Network extensibility scenario for flexible hybrid WDM/TDM PONs

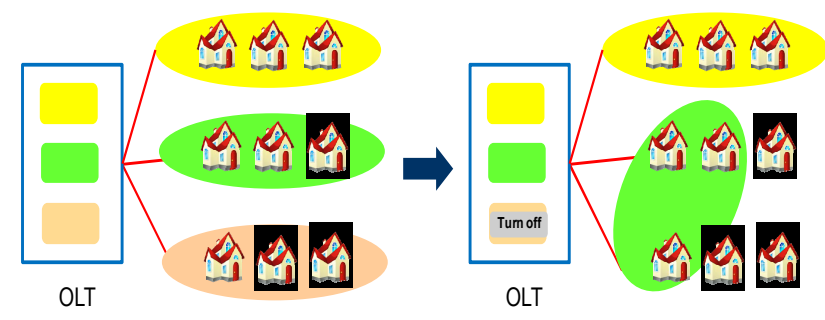

Figure 3. Energy efficiency scenario for flexible hybrid WDM/TDM PONs

\section{B. Energy Efficiency}

Our proposal for energy efficiency has a similar argument as provided for the network extensibility. An illustrative example is provided in Fig. 3 to demonstrate this. The left part of Fig. 3 shows that over time, users who require almost no services (e.g., business users during the night time hours) can be reconnected to a particular wavelength. In this scenario, the wavelength can be reallocated as shown in the right-hand part of Fig. 3 and this provides a mean to turn off some of the OLT transceivers as well as line cards, to enhance energy savings at the OLT over time. This might help the operator to build its network greener. The same scheme also depicts the possibility of dynamic allocation of wavelengths amongst users according to their traffic needs.

\section{Network Migration}

Our proposal for network migration corresponds to an efficient migration strategy that the proposed architecture can support during e.g. a technology upgrade. In this way, coexistence of a new technology with the legacy system is made possible. Fig. 4 provides an example where some subscribers move from a legacy $1 \mathrm{G}$ wavelength service to a new $10 \mathrm{G}$ wavelength service. In this case, by reallocation of wavelengths as shown in the right part of the Fig. 4, the network demands can be accomplished by just having one OLT transceiver that supports a $10 \mathrm{G}$ wavelength. This strategy allows a more smooth transition of the network during the migration phase.

\section{Constraints Raised by Enhanced Flexibility}

Adding extra flexibility in the access network comes also at the cost of other drawbacks, which have to be carefully tackled. The most important constraints are equipment cost, power budget which specifies the total optical power loss from the OLT to the ONUs, and security issues. The security issues generally come from the broadcast or multicast nature of the PON infrastructure. Therefore, PONs with a higher multicasting domain suffer from more severe security threats due to the sharing of the same media by more ONUs (users).

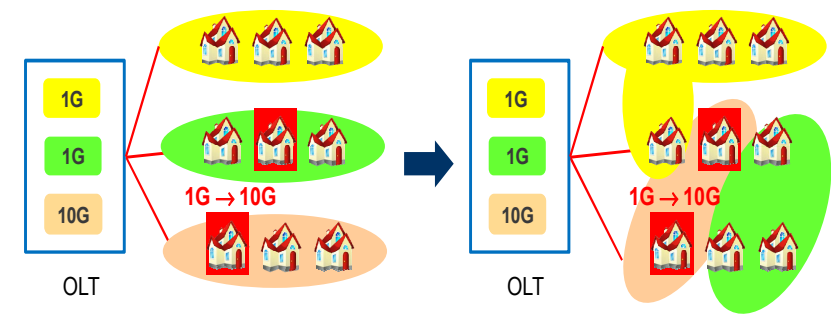

Figure 4. Migration scenario for flexible hybrid WDM/TDM PONs 


\section{EVALUATION OF FLEXIBILITY IN HYBRID WDM/TDM PONS}

As described in Section II, the proposed hybrid WDM/TDM PONs have a different degree of flexibility, going from fully static, over partially flexible to fully flexible architectures. An important assessment parameter of the flexibility is the number of needed wavelengths at a certain offered ONU load for the same PON performance like propagation delay of the packet from a user to the OLT, packet loss rate etc. For a fully flexible architecture, it is clear that the number of needed wavelengths can be optimally minimized, but how big is the gain of a fully flexible architecture compared to a partially flexible one, and what is the minimum degree of flexibility required to have a significant advantage of the offered flexibility. This section will give an answer to the above questions.

Although the flexibility is introduced by the architectural design, it is of importance to develop a well-suited MAC protocol that optimally exploits the offered flexibility in terms of dynamic wavelength allocation. We give the description of the MAC protocol used for the investigation of flexibility in hybrid WDM/TDM PONs. We also describe the various variants of hybrid WDM/TDM PON considered for the flexibility evaluation. Further, based on a basic simulation environment, we investigate the influence of flexibility on the wavelength usage and PON performance.

\section{A. MAC Protocols for Hybrid WDM/TDM PONs}

For the described architectures in Section II, a suitable MAC protocol is needed to manage the time and wavelength allocation and scheduling. The problem of bandwidth scheduling in hybrid WDM/TDM PONs can be approached in fundamentally two different ways [5]: a) Separated time and wavelength assignment, and b) Joint time and wavelength assignment. Joint time and wavelength assignment is a multidimensional scheduling approach which is more complex, but it is an efficient and scalable approach of upstream scheduling and wavelength assignment (USWA). There exist two USWA approaches [5]: offline and online. In the offline approach [6], the OLT waits until it has received all the reports from the ONUs (or part of them [7]) and then it performs some algorithm to find the best USWA scheme for the corresponding grants. In the online approach [8], upon the arrival and processing of a report from an ONU, the OLT immediately decides on the USWA for the corresponding grant.

For this paper, we have used an offline joint time and wavelength assignment scheme based MAC protocol, comparable to the protocol proposed in [9], to investigate the optimal degree of flexibility in hybrid WDM/TDM architectures. The offline approach has very less implementation complexity and can address fairness and QoS issues among different ONUs. In addition to this, in offline algorithms, the OLT would wait until the report messages from all ONUs have arrived and then try to arrange upstream scheduling in an optimal way, thus minimizing void formation, wavelength switching and wavelength use. Note that online algorithms can be useful for further optimizing the MAC protocol when very delay-sensitive applications are used, but for the purpose of this paper, the simpler offline approach completely fulfils our needs.

\section{B. Hybrid WDM/TDM PON Variants Used for Simulation}

We have simulated a hybrid WDM/TDM PON with $M$ $\left(=m_{A W G} \times m_{s}\right) \quad$ TDM PONs, consisting of $N$ ONUs (corresponding to a hybrid WDM/TDM PON with $M \times N$ ONUs). From the access side, packets arrive at the ONU from a user connected to that ONU. Packets are buffered in the ONU until the ONU is allowed to transmit them to the OLT. In our model, we consider $R_{U}$ to be the data rate of an upstream channel from an ONU to the OLT. We have chosen $M=16$, $N=4$, and $R_{U}=1 \mathrm{Gbps}$. We have generated packets in the form of Ethernet frames ( 64 to 1518 bytes) and packets arrive at each ONU from the end user. To reflect the property of Internet traffic, the generated user traffic is self-similar by aggregating 32 sub-streams each consisting of alternating Pareto-distributed on/off periods, with a shape parameter of 1.4 for the on period and 1.2 for the off period, as is done in previous studies [10]. In the on period, the packet arrival rate is exponentially distributed with the mean value of $A_{r}$ (in bps). We have used $A_{r}$ as $0.0125 \Phi$, where $\Phi$ is the TDM Load.

We have assumed that there are 16 wavelengths available for each OLT, and RN1 and RN2 provide a 1:M (1:16) split and a $1: N(1: 4)$ split, respectively, as shown in Fig. 5. The different architectures are evaluated for a partially flexible B\&S-PON combined with AWG, each with a varying degree of flexibility in RN1 by varying the values of $m_{A W G}$ and $m_{s}$, while keeping $m_{A W G} \times m_{s}=M$. For two extreme cases, this architecture is reduced to the broadcast-and-select PON architecture (with $m_{s}=16$ ) and the wavelength-split PON (with $m_{s}=1$ ), introduced in section II.A and II.B, respectively. The architecture with $m_{s}=16$ provides full flexibility and any of the subscribers can use any of the available 16 wavelengths. The architecture with $m_{s}=1$ has no flexibility, as all ONUs can only use their dedicated wavelengths. The flexibility gains are achieved due to the increased multicasting.

\section{Simulation Results}

We have simulated the five scenarios corresponding to the different value of $m_{A W G}$ and $m_{s}$, indicating by Group x. Group x indicates the number of TDM PONs $\left(\mathrm{x}=m_{s}\right)$ that can share the same wavelength, or the number of wavelengths that can be used by one TDM PON (or RN2). Fig. 6 and Fig. 7 shows the average and maximum number of wavelengths, respectively, required to satisfy the offered TDM PON (or RN2) load in the

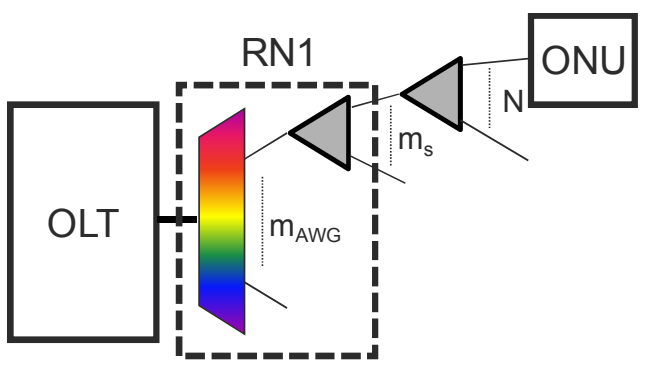

Figure 5. Hybrid WDM/TDM PON flavor based on AWG and power splitters considered for evaluation 
five considered scenarios. The TDM PON load is normalized with the load of $1 \mathrm{Gbps}$. We notice a faster increase of the average number of wavelengths for group 2 from a load of ca. 0.4 . This can be explained as for group 2, in the beginning, every TDM PON can typically be served by 1 wavelength, resulting in a total of 8 needed wavelengths. With higher loads, multiple (and not only one by one) TDM PONs will need a second wavelength, explaining the faster increase from that point. The simulation results clearly show that, already from the moment a certain degree of flexibility is available, large gains in terms of wavelength usage are possible, but from a given point the extra gain is very limited (the average number of wavelengths used from Group 4 to Group 16 is nearly the same). The less number of wavelengths used in a Group sharing a higher number of TDM PONs is due to the more efficient utilization of the wavelengths. Fig. 8 gives the channel under-utilization for the five considered groups.

To evaluate the TDM PON performance, we calculated the probability of a wavelength to be requested for three different load scenarios $(0.25,0.55$ and 0.95$)$. Fig. 9 and Fig. 10 give the probability of a certain number of wavelengths requested for

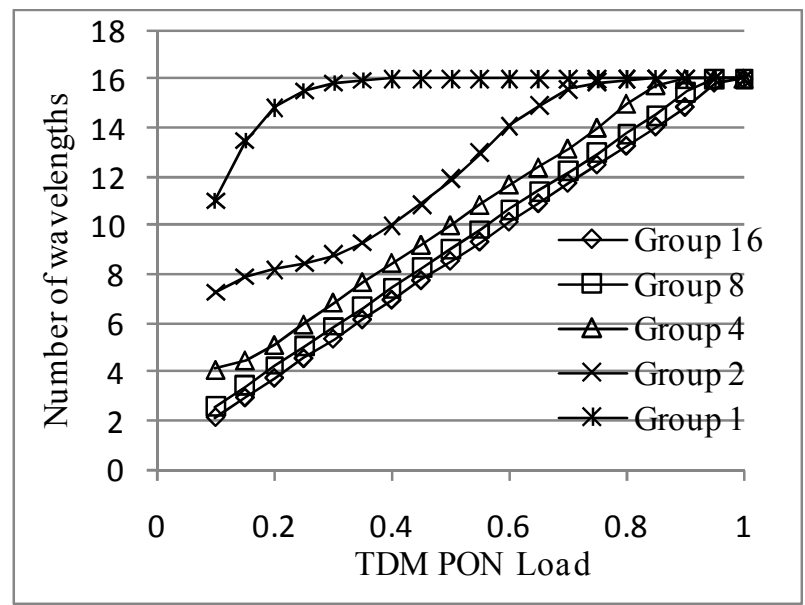

Figure 6. Average number of wavelengths required in function of the TDM PON or RN2 load, for five hybrid WDM/TDM PON variants with a different degree of flexibility in RN1

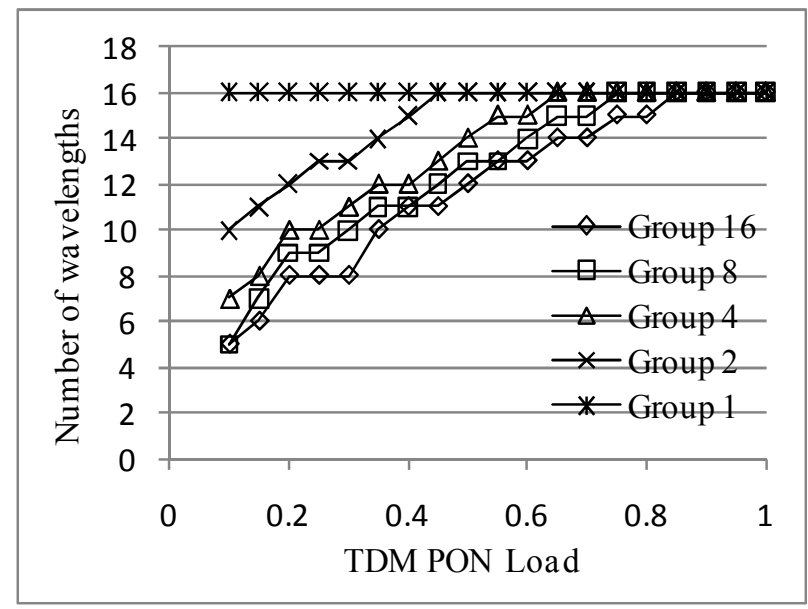

Figure 7. Maximum number of wavelengths required in function of the offered TDM PON or RN2 load, for five hybrid WDM/TDM PON variants with a different degree of flexibility in RN1 the three load scenarios, and this for Group 4 and 8 respectively. An AWG-based RN1 has the restriction that the number of wavelength allocated to a particular group cannot

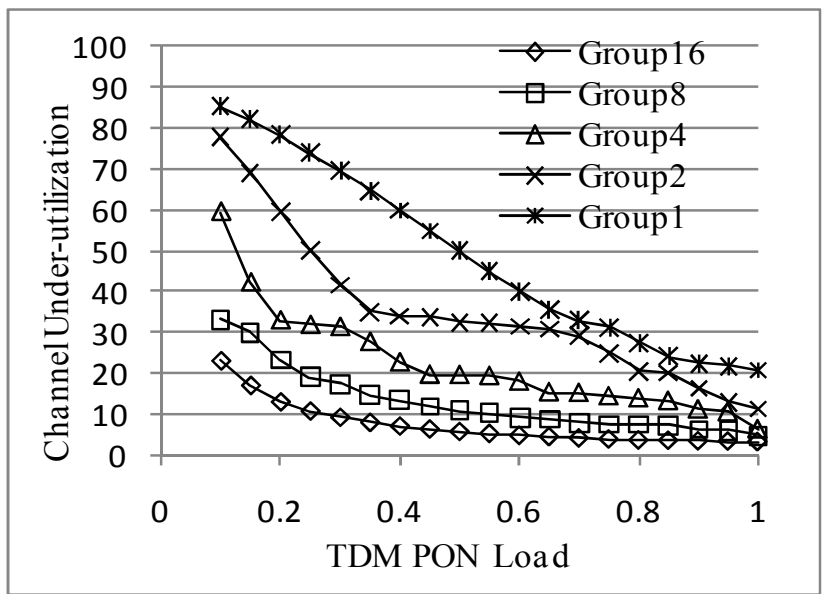

Figure 8. Channel under-utilization in function of the offered TDM PON or RN2 load, for five hybrid WDM/TDM PON variants with a different degree of flexibility in RN1

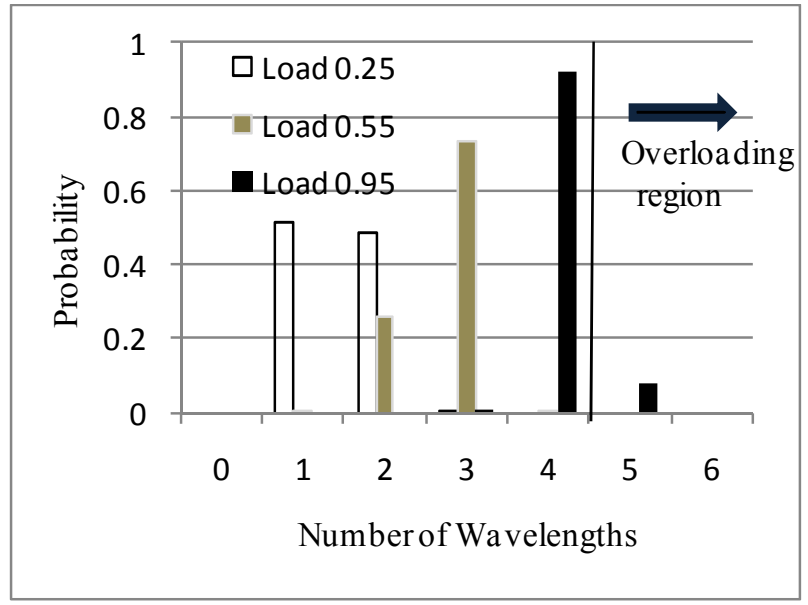

Figure 9. Probability of a certain number of wavelengths requested by a TDM PON for Group 4 and a TDM PON load of $0.25,0.55$ and 0.95 .

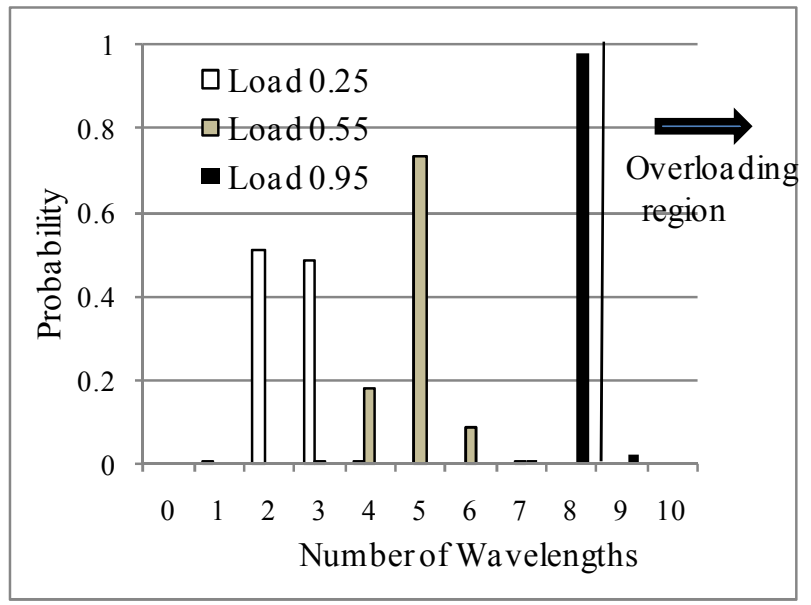

Figure 10. Probability of a certain number of wavelengths requested by a TDM PON for Group 8 and a TDM PON load of 0.25. 0.55 and 0.95 . 
exceed $m_{s}$ (i.e. Group number). Thus, the number of times a group demands for more wavelengths than the Group number, corresponds to an overloading situation as the channel capacity that is demanded is higher than the maximum channel capacity that can be allocated. The higher the probability that a Group falls in an overloaded region, the higher the PON performance degradation is. From Fig. 9 and Fig. 10, we can see that a TDM PON less frequently requests a number of wavelengths in the overloaded region for Group 8 than Group 4. From, the overloading probability of a TDM PON $\left(P^{g}\right)$, we can easily calculate the probability of over-loading of the entire network $\left(P^{n}\right)$ as $(1)$ :

$$
P^{n}=1-\left(1-P^{g}\right)^{m_{A W G}}
$$

Fig. 11 gives us the overloading probability of the PON network $\left(P^{n}\right)$ for three different loads. We see that as the group number increases, the probability of an overloaded situation decreases, since in a larger group base we have the flexibility to multicast more wavelengths to each TDM PON group. We refer this as multicasting gain. Note that though Group 4 and Group 8 use nearly the same number of average wavelengths, there will be a significant difference between the performances of both groups due to the considerable decrease of about $28 \%$ of an overloading situation in Group 8. We also see that there is no considerable difference in the Group performances when the offered TDM load is less than 0.55 . This result helps us to conclude that a partial flexible solution (with $m_{A W G} \geq 4$ ) gives us a comparable performance to a fully-flexible (with $m_{A W G}=$ 16) one in terms of the average number of wavelength usage, and it is an ideal situation if the expected TDM PON offered load is low. However, for a PON network serving critical customers or when the expected TDM PON offered load is high, the partial flexible solutions may not be the best situation.

\section{CONCLUSION}

Different hybrid WDM/TDM PON variants are discussed in this paper, and the main differences between them are highlighted. Special attention is given to flexible PON architectures, and we listed several flexibility advantages, like

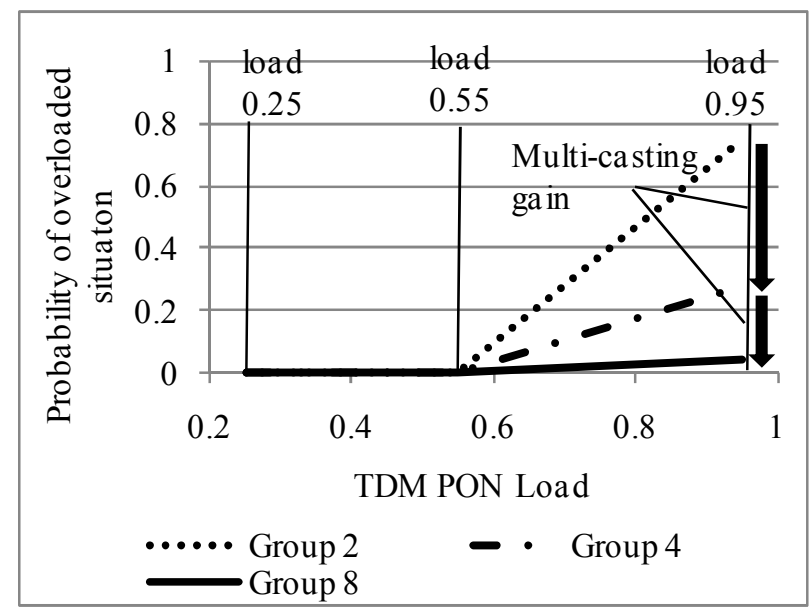

Figure 11. Probability of a overloaded situation in a TDM PON for a load of $0.25,0.55$ and 0.95 . energy efficiency, network migration and network extensibility. It is clear that there does not exist a one-fits-all solution that can offer high flexibility in combination with low cost, low power loss and high security. We further analyzed the average number of used wavelengths in a partially flexible remote node consisting of power splitters and AWGs. We have shown that from the moment a certain degree of flexibility is available, large gains in terms of wavelength usage are already possible, and beyond a certain point, additional flexibility does not provide much benefit in terms of wavelength consumption. We further analyzed the performance degradation (in terms of overloading probability). This study gives us insight in understanding the various parameters on which flexibility has to be evaluated. The future work may include the study of the flexibility effect on various performance parameters like propagation delay from the user to the OLT, packet loss rate etc. Moreover, other partially flexible architectures, e.g. using wavelength selective switches (WSS) [2], may be evaluated.

\section{ACKNOWLEDGMENT}

The research leading to these results has received funding from the European Community's Seventh Framework Programme (FP7/2007-2013) under grant agreements $\mathrm{n}^{\circ}$ 212352 (ICT-ALPHA) and $n^{\circ} 249025$ (ICT-OASE).

\section{REFERENCES}

[1] 249025-ICT OASE Project, Requirements for European NextGeneration Optical Access Networks, D2.1.

[2] G. Das, B. Lannoo, D. Colle, M. Pickavet, and P. Demeester, "A Hybrid WDM/TDM PON Architecture Using Wavelength Selective Switches", 4th IEEE International Symposium on Advanced Networks and Telecommunication Systems (ANTS 2010), Mumbai, India, Dec. 16-18, 2010 .

[3] C. Bock, J. Prat, and S. D. Walker, "Hybrid WDM/TDM PON Using the AWG FSR and Featuring Centralized Light Generation and Dynamic Bandwidth Allocation", Journal of Lightwave Technology, vol. 23, no. 12, Dec. 2005, pp. 3981-3988.

[4] N. Calabretta, M. Presi, R. Proietti, G. Contestabile, and E. Ciaramella, "A Bidirectional WDM/TDM-PON Using DPSK Downstream Signals and a Narrowband AWG", IEEE Photonics Technology Letters, vol. 19, no. 16, Aug. 2007, pp. 1227-1229.

[5] M. P. McGarry, M. Reisslein, "Bandwidth management for WDM EPONS," Journal of Optical Networking, Vol. 5, No. 9, September 2006.

[6] A. R. Dhaini, C. M. Assi, A. Shami, "Dynamic wavelength and bandwidth allocation in hybrid TDM/WDM EPON networks", Journal of light wave technology, Vol. 25, No. 1, January 2007.

[7] M. P. McGarry et al., "Just-in-time scheduling for multichannel EPONs,”J. Lightw. Technol., vol. 26, no. 10, pp. 1204-1216, May 2008.

[8] K. Kanonakis and I. Tomkos, "Improving the efficiency of online upstream scheduling and wavelength assignment in hybrid WDM/TDMA EPON networks," IEEE Journal on selected areas in Communications, Vol.28, No.6, August 2010.

[9] G. Das, B. Lannoo, H.-D. Jung, T. Koonen, D. Colle, M. Pickavet, and P. Demeester, "A New Architecture and MAC Protocol for Fully Flexible Hybrid WDM/TDM PON”, ECOC 2009, Sep. 2009, paper P6.28.

[10] W. Willinger, M. Taqqu, R. Sherman, and D. Wilson. "Self-similarity through highvariability: statistical analysis of Ethernet LAN traffic at the source level", Proc., ACM SIGCOMM '95, pp. 100-113, Cambridge, MA, August 1995. 